\title{
Radiation damage yields across the carbon 1s excitation edge
}

\author{
Adam F.G. Leontowich ${ }^{1 \neq^{*}}$, Adam P. Hitchcock ${ }^{1}$ and Ray F. Egerton ${ }^{2}$
}

1. Brockhouse Institute for Materials Research, McMaster University, Hamilton, L8S 4M1 Canada

2. Department of Physics, University of Alberta, Edmonton, T6G 2E1 Canada ABSTRACT: X-rays are used as a probe in many materials analysis techniques, but they may induce changes in the sample that can invalidate the measurement. Strategies that overcome "radiation damage" are therefore desirable. Tuning the incident photon energy below a core electron excitation edge of a material (where only valence excitation takes place) rather than above it (where primarily core excitation occurs) has been theorized as a way to reduce radiation damage. To investigate this strategy, we used a scanning transmission x-ray microscope to expose thin films of organic polymers to x-rays with photon energies below and above the $\mathrm{C} 1 \mathrm{~s}$ excitation edge. The extent of radiation damage was quantified by $\mathrm{x}$-ray absorption spectroscopy and lithography. We found that the amount of radiation damage per unit absorbed dose was the same using incident photons below and above the $\mathrm{C}$ 1s excitation edge. We conclude that the turn on of Auger processes when $\mathrm{C}$ 1s holes are created does not affect the overall radiation damage outcome.

KEYWORDS: K edge effect, core edge effect, radiation damage, STXM, x-ray lithography.

‡Corresponding author: Tel: 1-306-657-3555, Email: adam.leontowich@lightsource.ca *Present address: Canadian Light Source Inc., Saskatoon, S7N 2V3 Canada 


\section{INTRODUCTION}

Many materials analysis techniques, such as electron microscopy, x-ray crystallography and x-ray spectroscopy, use ionizing radiation to probe materials. For these techniques, the absorbed dose, in Système International units of Grays where $1 \mathrm{~Gy}=\mathrm{J} \mathrm{kg}^{-1}$, is proportional to the sample exposure time i.e. the dwell time, and the x-ray absorption coefficient of the material. Higher doses improve the signal to noise ratio and, by providing better contrast, also improve the effective spatial resolution $[1,2]$. Ideally, it would be beneficial to probe samples with high doses to get data of the highest quality and spatial resolution. However, materials often experience physical and chemical changes when exposed to ionizing radiation, and the magnitude of these changes are linked to the dose. The rates of change versus dose happen to be high for most organic matter, except for aromatic species $[3,4]$. The effects of ionizing radiation on many organic materials have been well characterized by electron microscopy [5-7], x-ray crystallography [8] and x-ray spectromicroscopy [9]. For example, the dose-related effects associated with the acquisition of an electron energy loss spectrum may result in a spectrum which is not that of the intended virgin specimen, but rather a sum of the virgin specimen, the cinder of the specimen, a beam-deposited carbon contamination layer [10] and other effects. Radiation-induced changes to materials are generally referred to as "radiation damage" in situations where they are perceived to be undesirable.

A great deal of effort has been spent understanding radiation damage, and this research has led some to develop strategies to overcome it. These strategies share common themes. One theme is to avoid high dose - simply keep the dose below an established level at which the data, relevant to a particular application, starts to be significantly 
compromised by radiation damage $[2,5,11]$. This alone may not be enough to collect a data set of sufficient quality, so another more proactive theme is to preserve the original state of the sample at high doses. The most widely implemented strategy in this regard has been sample cooling, which is particularly effective in macromolecular x-ray crystallography. The lifetime of a sample with respect to diffraction contrast is typically increased by one to two orders of magnitude by cooling to liquid nitrogen temperatures or "cryo-cooling" ( $100 \mathrm{~K})$ [8]. The mechanism of preservation is a cage effect whereby reactive and/or volatile radiolysis products are essentially frozen in place. When a cryocooled, irradiated sample is allowed to warm up to room temperature, the amount of damage observed is approximately what would have been incurred without cooling $[12,13]$. Yet another theme is to sacrifice the individual sample, by collecting data faster than the damage processes propagate, and/or combining data from multiple sample copies, if available. For example, serial femtosecond crystallography relies on the principle of "diffraction before destruction" $[14,15]$. Here, although the battle to preserve individual samples is lost, while the war of collecting high dose data, free of radiation damage artefacts, is won.

Johnson and Isaacson studied radiation damage of nucleic acid bases using electron energy loss spectroscopy as Ph.D. students of Albert Crewe at the University of Chicago. Both concluded that their observations were consistent with a model in which radiation damage processes in organic matter are primarily induced by core electron (1s or K-shell in this case) excitation (99\% [16]), and that lower energy valence electron excitation does not result in radiation damage [16,17]. Isaacson later refined his view, and suggested that valence excitation can induce radiation damage in non-conjugated aliphatic moieties 
while 1s excitation is necessary to damage aromatic moieties [4]. Durup and Platzman analysed the processes which follow 1s excitation in hydrocarbons and proteins, and theorized a significant increase in radiation damage yields for 1s excitation versus valence excitation [18]. Cosslett suggested that "inner-shell excitations [which result in Auger events] may contribute more to radiation damage" [5]. For an element such as C, excitation with an incident energy which is less than the 1s excitation energy (the onset of which is usually between 280 and $290 \mathrm{eV}$, dependent on the chemical environment [19]) causes only valence excitation. Ionized states that are formed below a core edge tend to have a lower fraction of dissociative decay pathways [20]. In contrast, multi-electron Auger decay processes become dominant when the excitation energy is greater than a core excitation energy. The final charge state after Auger decay is at least doubly charged, which leads to bond breakage in almost all cases [20]. If it is indeed the case that irradiating with an incident energy which is less than a core excitation results in a net reduction in the amount of damage for a given dose, then using a photon energy below that core excitation threshold could be exploited as a radiation damage reduction strategy to preserve the original state of the sample to high doses. Other incident energy thresholds have been confirmed by experiment. For example, photons with energies below $4.7 \mathrm{eV}$ do not damage poly(methyl methacrylate) (PMMA) at low intensity [21]. There are also many compound-dependent energy thresholds for atomic displacement or "knock-on" damage in electron microscopy [6,22]. A core excitation threshold could be of practical use for crystallography, scattering, lithography, etc., in situations where the choice of incident energy is not critical to the physics of the experiment. 
The existence of an incident energy threshold for radiation damage, rooted in valence versus core electron excitation, is the subject of this paper. There have been several investigations of this damage threshold, which has sometimes been termed a "core edge effect," or a "K-edge effect". For example, a two-fold reduction in the death of V79 Chinese hamster cells was found when irradiating with $\mathrm{x}$-rays at $250 \mathrm{eV}$ versus $340 \mathrm{eV}$ (below and above the $\mathrm{C}$ 1s edge, respectively), and the difference was attributed to valence versus core excitation [23]. In contrast, Goodhead found that x-rays of $277 \mathrm{eV}$ (below the $\mathrm{C} 1 \mathrm{~s}$ edge) induced more inactivation and cell mutations in Chinese hamster cells, and more inactivation of human diploid fibroblasts than other photon energies above the $\mathrm{C}$ 1s edge at equivalent dose [24]. Kobayashi et al. irradiated yeast cells at the P 1s edge and found increased cell inactivation on the resonant absorption peak compared to off-peak radiation [25]. Chen et al. irradiated yeast cells below and above the $\mathrm{O} 1 \mathrm{~s}$ edge, and found that cell death was proportional to dose, and independent of photon energy [26]. Some core edge effects have been observed in aqueous solutions [27]. Several investigations have been carried out using x-ray crystallography. Most of these studies reported no difference in damage yields comparing valence versus core excitation over a broad photon energy range of $6.5 \mathrm{keV}$ to $33.0 \mathrm{keV}$ (Ref. [28], and references therein). However, the samples and/or conditions used in the studies cited in this paragraph were not optimal to determine whether the differences observed resulted only from valence versus core excitation, due to the presence of water and/or air during irradiation which are notorious for affecting radiation chemistry [29]. Dose determination in hydrated systems is difficult because radical migration causes the volume of material affected by radiation to be larger than the physical dimensions of the irradiated volume. 
The absorption sites in cells and protein crystals are heterogeneously distributed, leading to preferential absorption in certain spatial regions at certain photon energies.

Some core edge effect investigations have used more ideal samples: homogeneous thin solid films, irradiated in vacuum or an inert atmosphere. Such conditions are necessary to properly study the threshold mechanism in question. Yokoya et al. prepared thin films of DNA and measured strand breakage induced by $388 \mathrm{eV}$ and $435 \mathrm{eV}$ photons (below and above the $\mathrm{N}$ 1s edge, respectively). Strand breakage did not depend on photon energy [30]. Fujii and Yokoya irradiated DNA films below $(395,528 \mathrm{eV})$ and above $(408,538$ $\mathrm{eV}$ ) the $\mathrm{N}$ and $\mathrm{O} 1 \mathrm{~s}$ edges, respectively, and observed a difference in the $\mathrm{O} 1 \mathrm{~s}$ near edge x-ray absorption fine structure (NEXAFS) spectrum of the film irradiated at $538 \mathrm{eV}$ [31]. Wang et al. irradiated polyethylene terephthalate at several photon energies below, on and above the $\mathrm{O} 1 \mathrm{~s}$ edge. No differences were observed in the O 1s NEXAFS spectra of the irradiated material beyond the measurement uncertainty, and the net radiation damage yields were found to be independent of photon energy [32]. Hieda et al. irradiated thin films of DNA at several photon energies around the P 1s edge and found that single strand breaks were dependent on dose and independent of photon energy, while slightly more double-strand breaks occurred for a given dose when the excitation energy was above the $\mathrm{P}$ 1s edge [33].

The $\mathrm{C} 1 \mathrm{~s} \mathrm{x}$-ray absorption cross section is a non-negligible component of the total $\mathrm{x}$-ray absorption cross section of organic matter at the $\mathrm{N} 1 \mathrm{~s}, \mathrm{O}$ 1s, or P 1s edges. This means that excitation with an incident energy just below the $\mathrm{O} 1 \mathrm{~s}$ edge for example will result in valence excitation of $\mathrm{O}$, but it will also result in some core excitation of $\mathrm{C}$. According to the hypothesized threshold mechanism, the dependence on excitation energy should be 
most pronounced at the $\mathrm{C} 1 \mathrm{~s}$ edge, because there are no underlying core ionization contributions, at least for species consisting of solely H-C-N-O. Therefore, for organic matter consisting of $\mathrm{H}-\mathrm{C}-\mathrm{N}-\mathrm{O}$, the theorized difference in radiation damage yields rooted in valence versus core electron excitation should be maximized at the $\mathrm{C}$ 1s edge. Excitation with an incident energy below the $\mathrm{C}$ 1s edge would result in exclusively valence excitation of all elements, while for incident energies just above the $\mathrm{C} 1 \mathrm{~s}$ edge, only $\mathrm{C}$ will experience $1 \mathrm{~s}$ excitation. The relative yields of fragment ions of polystyrene [34] and PMMA [35,36] have been shown to depend on photon energy across the C $1 \mathrm{~s}$ edge. However, the signal for damage in those measurements was the ions that happened to desorb from the sample surface, and the changes in the sample itself were not measured. Howie et al. [37] and Stephens et al. [38] searched for this threshold by irradiating highly conjugated organic molecules with low energy electrons. Both observed an energy threshold for damage in the vicinity of the $\mathrm{C} 1 \mathrm{~s}$ edge. However, electrons are not the optimal radiation to study the hypothesized threshold effect because; 1) at low incident kinetic energies $(<1000 \mathrm{eV})$, the penetration depth is only a few nanometers, and 2) excitation via inelastic electron scattering is not selective - many more valence excitations will occur relative to the desired core events.

The goal of this study was to investigate the hypothesized incident energy threshold for radiation damage. This required careful experiment design because the quantitative characteristics of radiation damage are critically dependent on the small details, such as the presence of air, water, dose rate, temperature, carbon contamination and so on. We quantified and/or eliminated these potential sources of error, and reduced this question down to experiments with a single variable - incident photon energy - performed at the C 
1s edge. This is the most likely energy region to observe an incident energy threshold for radiation damage rooted in valence versus core electron excitation for organic matter.

\section{EXPERIMENTAL}

\subsection{Sample preparation}

Poly(methyl methacrylate) (PMMA, electronics grade, $M_{\mathrm{w}}=315000 \mathrm{~g} / \mathrm{mol}, M_{\mathrm{w}} / M_{\mathrm{n}}=$ $1.05)$ and polystyrene (PS, $\left.M_{\mathrm{w}}=104000 \mathrm{~g} / \mathrm{mol}, M_{\mathrm{w}} / M_{\mathrm{n}}=1.05\right)$ were purchased from Polymer Source, Inc. and used as received. Thin films of PMMA or PS were fabricated by spin casting solutions of polymer in toluene (99.9\% Chromasolv®, Sigma-Aldrich) onto freshly cleaved $15 \mathrm{~mm} \times 15 \mathrm{~mm}$ pieces of mica (Ted Pella, Inc.). $2 \mathrm{~mm} \times 2 \mathrm{~mm}$ thin film pieces were transferred onto $75 \mathrm{~nm} \mathrm{SiN}$ windows (nominally $\mathrm{Si}_{3} \mathrm{~N}_{4}$, Norcada, Inc.) or uncoated $\mathrm{Cu}$ transmission electron microscopy (TEM) grids (Ted Pella, Inc.) by a float method [39]. The films were then annealed at 20 mTorr for $1 \mathrm{~h}$ to remove residual solvent and improve film uniformity. The anneal temperature was set at $423 \mathrm{~K}$ for PMMA and $413 \mathrm{~K}$ for PS, which is above the glass transition temperature but below the degradation temperature for both polymers in this thickness range $[39,40]$. Film thicknesses were determined after annealing using a Quesant Q-Scope 350 atomic force microscope (AFM) equipped with Budget Sensors Multi75Al cantilevers (tip radius: $<10$ $\mathrm{nm}$ ), at a scan rate of $0.5 \mathrm{~Hz}$ in intermittent contact mode, and using C 1s NEXAFS spectra normalized to $1 \mathrm{~nm}$ of material [39]. The PMMA films were $50 \pm 3 \mathrm{~nm}$ thick, while the PS films were $40 \pm 3$ nm thick. 


\subsection{X-ray exposure}

The Polymer STXM [41] at beamline 5.3.2.2, Advanced Light Source (ALS, Berkeley, USA), and the Ambient STXM at beamline 10ID-1 [42], Canadian Light Source (CLS, Saskatoon, Canada) were used to expose samples to monochromatic x-rays in a controlled manner, and also to collect images and C 1s NEXAFS spectra from exposed and unexposed sample regions. The Fresnel zone plate lenses used were obtained from the Center for X-ray Optics (CXRO), LBNL (240 $\mu \mathrm{m}$ diameter, $95 \mu \mathrm{m}$ central stop, 25 $\mathrm{nm}$ outer most zone width). Low pass filters (a differentially pumped, $1.0 \mathrm{~m}$ long section of the beamline filled with 600 mTorr $\mathrm{N}_{2}$ at 5.3.2.2; a $200 \mathrm{~nm}$ thick Ti foil at 10ID-1) in combination with a properly aligned order-sorting aperture and central stop, were used to suppress all radiation other than the beamline first order, zone plate +1 order to negligible levels. The monochromator entrance and exit slit widths were chosen to exceed the diffraction-limited performance of the zone plate in terms of both temporal and spatial coherence across the entire photon energy region investigated. The photon energy resolution was $0.1 \mathrm{eV}$ and the spatial resolution (Rayleigh criterion) was $30 \mathrm{~nm}$ under these conditions. The efficiency of the scintillator-photomultiplier tube detector was measured at both STXMs before the experiments began [39]. After loading the samples, the STXM vacuum chamber was evacuated and then backfilled with 250 Torr He. We have previously reported full details of STXM imaging, NEXAFS spectroscopy and controlled x-ray exposure using a pattern generation program elsewhere [39].

The dose calculation method has also been reported in detail elsewhere [39] and the dose accuracy is estimated as $10 \%$. The calculation accounts for changes in the $\mathrm{x}$-ray absorption cross section of the sample with dose due to mass loss over the course of the 
exposure. The area irradiated was controlled using a pattern generation program with positional feedback from a laser interferometer [41]. It is also important to consider whether the irradiated volume actually contains all of the energy absorbed. As the photon energy increases, a greater portion of energy initially deposited will escape from the irradiated volume by means of secondary effects, such as large inelastic mean free paths for the photoelectrons and $\mathrm{x}$-ray fluorescence, especially for higher $Z$ elements [43-45]. These effects could skew the dose calculation and thus the core edge effect, if left unchecked. For electrons generated by $\mathrm{x}$-rays in the $\mathrm{C} 1 \mathrm{~s}$ edge region, the electron inelastic mean free path is less than $3 \mathrm{~nm}$ [46], and the probability of a core hole being filled by a radiative process is very small relative to an Auger process [47]. Therefore, we assume that all of the energy deposited in the sample remains within $10 \mathrm{~nm}$ of the irradiated volume.

To test for the presence of an effect associated with irradiating below rather than above the $\mathrm{C}$ 1s edge, exposures must be made at constant dose but with varying photon energy. Two things must be considered to achieve this condition: 1) the $\mathrm{x}$-ray absorption cross sections of $\mathrm{C}$ containing materials change drastically with photon energy across the $\mathrm{C} 1 \mathrm{~s}$ edge (this can be directly quantified by collecting the C 1s NEXAFS spectrum of the material), and 2) the incident x-ray flux of the STXMs, measured downstream of the aligned zone plate and order-sorting aperture, vary significantly across the $\mathrm{C} 1 \mathrm{~s}$ edge. This is due in part to beamline design and in part to carbon contamination on optics [10]. There were two options to produce the desired iso-dose exposures. The first was to use a constant dwell time for the exposures, and adjust the monochromator exit slit widths and therefore the incident flux for each exposure in step with the x-ray absorption cross 
section of the sample. For this option, the dose rate would be held constant for all exposures, but the photon energy resolution and spot size would not be constant $[41,42]$. The second option was to hold the monochromator exit slit widths constant, and adjust the dwell time for each exposure in step with the x-ray absorption cross section of the sample and beamline flux output. For this option, the photon energy resolution and spot size would be held constant for all exposures, but the iso-dose exposures would experience different dose rates at different exposure photon energies. We chose the second option: hold the widths of the monochromator exit slits constant for all exposures. In a separate experiment using identical PMMA samples, we found that the amount of radiation damage, judged by the amount of $\mathrm{C} 1 \mathrm{~s}$ NEXAFS spectral change, was independent of dose rate over a larger dose rate range $(73-1230 \mathrm{MGy} / \mathrm{s})$ than was investigated here [39]. In another experiment, we found the rise in temperature at the focal point of the STXM was less than $1 \mathrm{~K}$ for dose rates up to $2.7 \mathrm{GGy} / \mathrm{s}$ [48].

\subsection{Lithographic development}

Some samples underwent a development procedure after exposure. PMMA samples were held with locking tweezers, stirred in 3:1 v/v 2-propanol (99.5\%, Caledon Laboratories): 4-methyl-2-pentanone (>98.5\% ACS reagent grade, Sigma-Aldrich) for $30 \mathrm{~s}$, then immediately stirred in 2-propanol for $15 \mathrm{~s}$ [49]. PS samples were held with locking tweezers, and stirred in xylenes (>98.5\% ACS reagent grade, Sigma-Aldrich) for $30 \mathrm{~s}$ $[49,50]$. The samples were allowed to dry in air after development, which was performed at $293 \pm 2 \mathrm{~K}$. 


\section{RESULTS}

\subsection{Poly(methyl methacrylate)}

The C 1s NEXAFS spectrum of a PMMA sample was measured and is presented in Fig. 1.

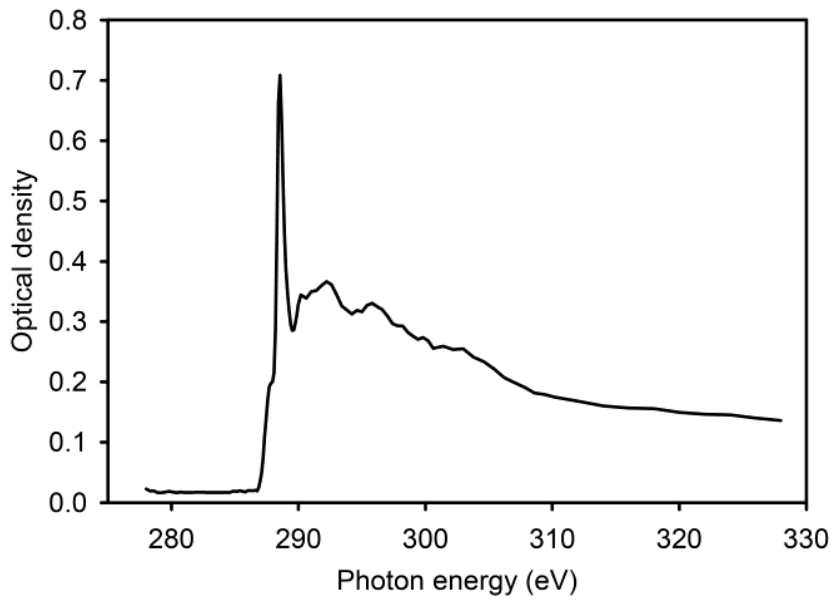

Figure 1: C 1s NEXAFS spectrum of a $50 \pm 3 \mathrm{~nm}$ thick film of PMMA.

A dose of $5 \mathrm{MGy}$ was used to acquire this spectrum. The prominent absorption feature at $288.4 \mathrm{eV}$ is the $\mathrm{C} 1 \mathrm{~s}(\mathrm{C}=\mathrm{O}) \rightarrow \pi^{*} \mathrm{C}=\mathrm{O}$ transition, localized on the carbonyl $\mathrm{C}$ atom of the PMMA side chain. Several reports have discussed this and other NEXAFS spectral features of PMMA and its radiation chemistry in detail $[9,13,29]$. As PMMA receives more dose, the feature at $288.4 \mathrm{eV}$ diminishes by exponential decay due to a net loss of $\mathrm{C}=\mathrm{O}$ bonds until reaching a residual value $[9,13,29,39]$. This signal is proportional to the number of $\mathrm{C}=\mathrm{O}$ bonds within the volume of film investigated, and can therefore be used as a quantitative indicator of radiation damage.

Nine $600 \mathrm{~nm} \times 600 \mathrm{~nm}$ areas of a PMMA on SiN sample were irradiated at $275.0 \mathrm{eV}$ (below the $\mathrm{C} 1 \mathrm{~s}$ edge), $288.4 \mathrm{eV}$ (on the $\mathrm{C} 1 \mathrm{~s}(\mathrm{C}=\mathrm{O}) \rightarrow \pi^{*} \mathrm{C}=\mathrm{O}$ transition) and $300.0 \mathrm{eV}$ (above the $\mathrm{C} 1 \mathrm{~s}$ edge) over a range of doses. The adsorbed dose rates for these exposures 
varied between $30-595 \mathrm{MGy} / \mathrm{s}$. The irradiated sample areas were imaged at $288.4 \mathrm{eV}$ immediately after patterning (Fig. 2(a)).

(a)
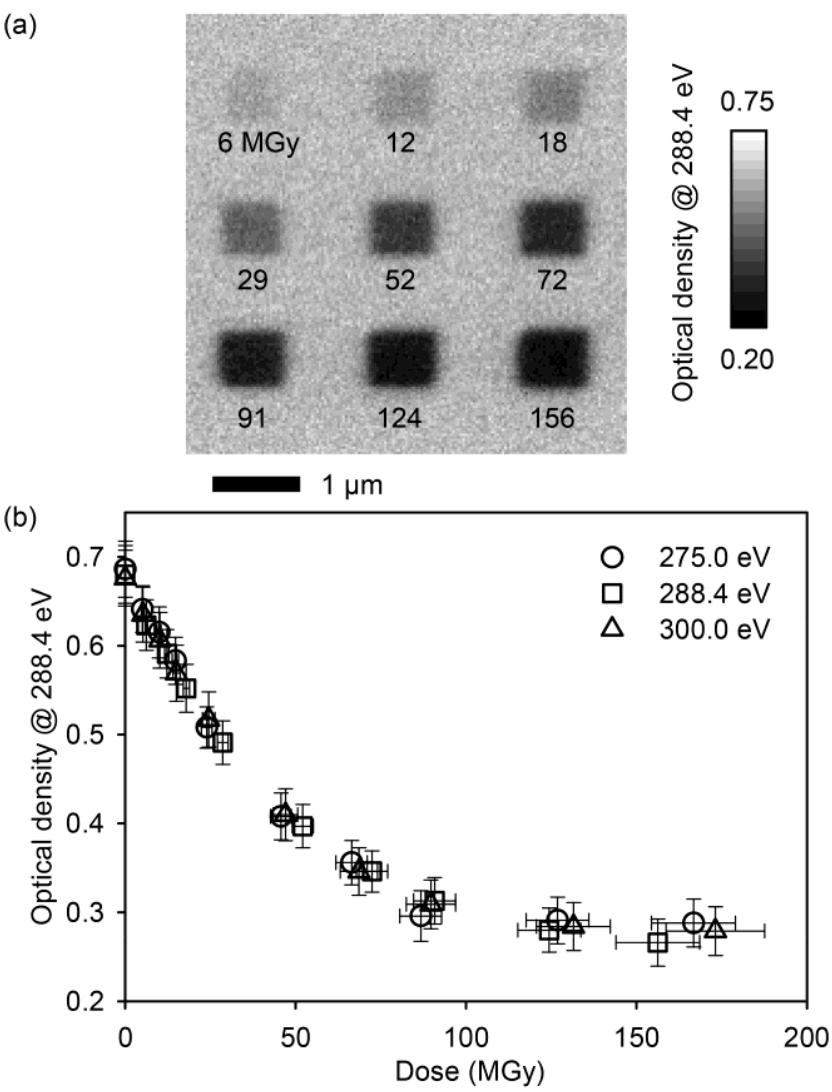

Figure 2: (a) Transmission x-ray micrograph, acquired at $288.4 \mathrm{eV}(\mathrm{C} 1 \mathrm{~s}(\mathrm{C}=0) \rightarrow \pi * \mathrm{C}=0$ peak) and converted to optical density, depicting nine areas of a $50 \pm 3 \mathrm{~nm}$ thick PMMA sample that were exposed to variable doses (values indicated). (b) Optical density at $288.4 \mathrm{eV}$ of irradiated areas of the sample plotted versus dose. Exposure photon energy: •, $275.0 \mathrm{eV}$; , $288.4 \mathrm{eV}$; $\triangle$, $300.0 \mathrm{eV}$.

The dose used to acquire each image was 1 MGy. The average optical density at 288.4 $\mathrm{eV}$ of each irradiated area was determined from these images and plotted versus dose (Fig. 2(b)). The rate of the decay of the $288.4 \mathrm{eV} 1 \mathrm{~s}(\mathrm{C}=\mathrm{O}) \rightarrow \pi^{*} \mathrm{C}=\mathrm{O}$ feature followed first-order kinetics as observed previously $[9,13,29,39]$. More importantly, 1) the amount of $\mathrm{C}=\mathrm{O}$ bond loss was found to be independent of photon energy at every dose level investigated, 2) the critical dose for $\mathrm{C}=\mathrm{O}$ bond loss for all three exposure energies was not statistically different from previous measurements (62.8 \pm 8 MGy [39]), and 3) 
identical results were obtained using a PMMA on TEM grid sample (i.e., freestanding film, no substrate), indicating negligible overall contributions from the SiN substrate.

In a second experiment, $600 \mathrm{~nm} \times 600 \mathrm{~nm}$ areas of a PMMA on SiN sample were irradiated at the same three photon energies, $275.0 \mathrm{eV}, 288.4 \mathrm{eV}$ and $300.0 \mathrm{eV}$, over a range of doses. The adsorbed dose rates for these exposures varied between $51-598$ MGy/s. For this experiment, the irradiated sample areas were not imaged by STXM after exposure. The sample was removed from the STXM vacuum chamber and underwent the PMMA development procedure (\$2.3). The developed irradiated areas were then imaged using AFM (Fig. 3). 
(a) $275.0 \mathrm{eV}$

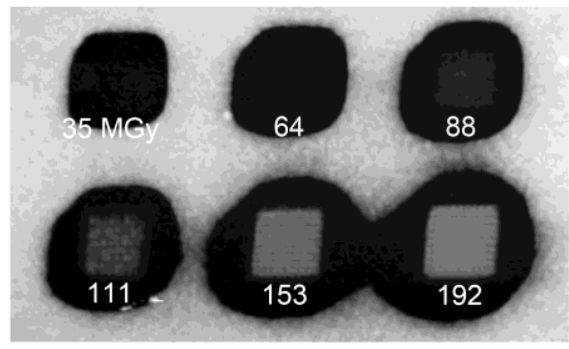

(b) $288.4 \mathrm{eV}$
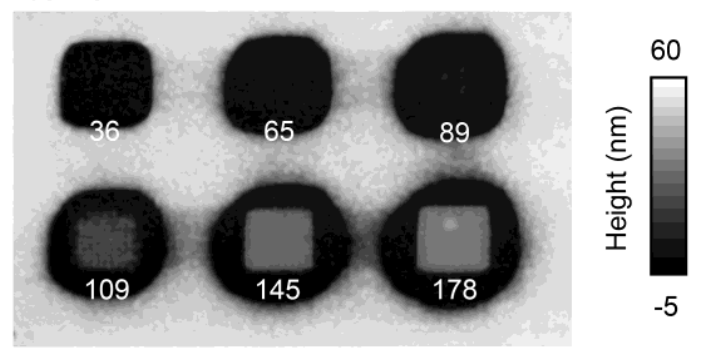

(c) $300.0 \mathrm{eV}$

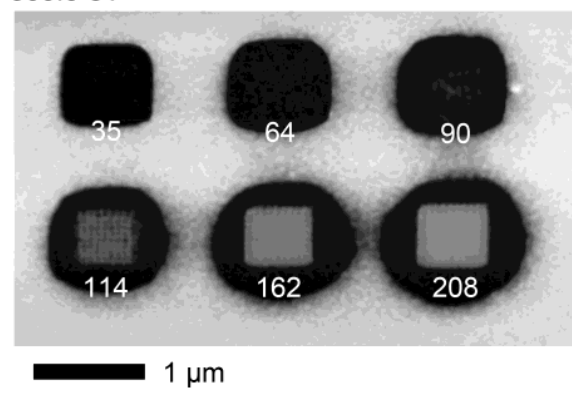

Figure 3: Atomic force micrographs of irradiated areas of a $50 \pm 3 \mathrm{~nm}$ thick PMMA sample after development. Exposure photon energy: (a), $275.0 \mathrm{eV}$; (b), $288.4 \mathrm{eV}$; (c), 300.0 eV. All micrographs are on the same height and spatial scale.

We had previously established that a lithographic transition from positive mode (i.e., the developer removes the irradiated polymer, leaving behind the non-irradiated material) to negative mode (i.e., the developer removes the non-irradiated polymer, leaving behind the irradiated material) was dose dependent and occurred at $90 \pm 5 \mathrm{MGy}$ at $300.0 \mathrm{eV}$ [51]. In this experiment, the onset of negative mode was always observed to occur at $90 \pm 5$ MGy, independent of photon energy.

One photon energy-dependent effect was noted in these lithography-based results. The morphology of the barely cross-linked PMMA area at $275.0 \mathrm{eV}$ (88 MGy, Fig. 3(a)) 
appeared more even and homogeneous than those at $288.4 \mathrm{eV}$ (89 MGy, Fig. 3(b)) or $300.0 \mathrm{eV}$ (90 MGy, Fig. 3(c)). We hypothesize that this observation arises from photoelectrons generated in the SiN substrate. The x-ray absorption cross section of PMMA is very low at $275.0 \mathrm{eV}$ relative to $288.4 \mathrm{eV}$ or $300.0 \mathrm{eV}$ (Fig. 1), therefore we must use more photons (in proportion to the C 1s NEXAFS spectrum of PMMA, Fig. 1) to achieve the same dose at this photon energy. However, the x-ray absorption cross section of $\mathrm{SiN}$ in the $\mathrm{C}$ 1s edge region is featureless and nearly flat, thus for iso-dose exposures, the number of photoelectrons emitted from the SiN surface at $275.0 \mathrm{eV}$ will be greater than at the other two energies. But this extra substrate contribution to radiation damage is only relevant within the electron escape depth (1/e $\sim 4 \mathrm{~nm}$ [9]). This explains why a substrate effect was observed for the barely crosslinked material at $90 \pm 5 \mathrm{MGy}$, but not observed for the next dose areas around and above 110 MGy (Fig. 3), because the height of the cross-linked material becomes much greater than the escape depth of secondary electrons generated in the substrate.

\subsection{Polystyrene}

The $\mathrm{C}$ 1s NEXAFS spectrum of a PS sample was measured and is presented in Fig. 4. 


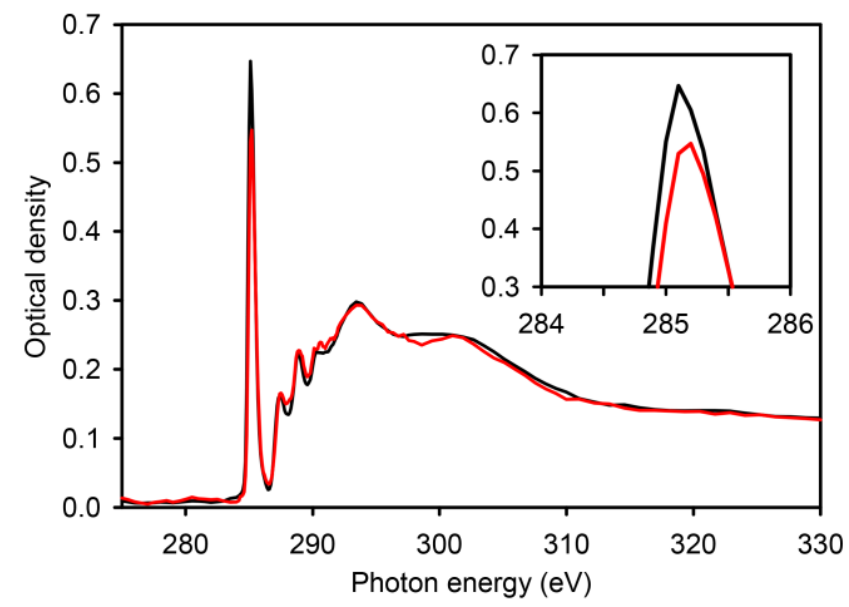

Figure 4: C 1s NEXAFS spectrum of a $40 \pm 3 \mathrm{~nm}$ thick film of PS (black), and after receiving $70 \mathrm{MGy}$ at $275.0 \mathrm{eV}$ (red). The inset shows the reduction and slight blue shift in the $\mathrm{C} 1 \mathrm{~s}(\mathrm{C}=\mathrm{C}) \rightarrow \pi^{*} \mathrm{C}=\mathrm{C}$ peak due to the 70 MGy exposure.

A dose of 7 MGy was used to acquire this spectrum. The prominent absorption feature at $285.1 \mathrm{eV}$ is the $\mathrm{C} 1 \mathrm{~s}(\mathrm{C}=\mathrm{C}) \rightarrow \pi^{*}(\mathrm{C}=\mathrm{C})$ transition, delocalized within the PS phenyl ring side chain. This and other C 1s NEXAFS absorption features of PS and their response to radiation have been reported previously [9,29]. As PS receives more dose, the feature at $285.1 \mathrm{eV}$ diminishes by exponential decay until reaching a residual value [9]. This signal is proportional to the number of $\mathrm{C}=\mathrm{C}$ bonds within the volume of film investigated, and can therefore be used as a quantitative indicator of radiation damage.

Previous reports have claimed that aromatic moieties cannot be damaged when the energy of the incident radiation is below the $\mathrm{C}$ 1s edge, and that the Auger process is needed to break the aromaticity, i.e., that damage is mostly $[18,38]$ or entirely $[16,17]$ induced by $\mathrm{C} 1 \mathrm{~s}$ excitation. To investigate, a $600 \mathrm{~nm} \times 600 \mathrm{~nm}$ area of a PS on TEM grid sample (i.e., freestanding film, no substrate) was irradiated at $275.0 \mathrm{eV}$ to a dose of 70 MGy. Then, a C 1s NEXAFS spectrum of the irradiated area was measured (Fig. 4). A dose of 7 MGy was used to acquire the spectrum. The spectrum shows a reduction in the 
$\mathrm{C} 1 \mathrm{~s}(\mathrm{C}=\mathrm{C}) \rightarrow \pi^{*}(\mathrm{C}=\mathrm{C})$ peak, and a slight blue shift compared to that of virgin PS. This signifies the loss of $\mathrm{C}=\mathrm{C}$ bonds, present only in the phenyl ring. Thus we find PS can indeed be damaged by valence excitation using x-rays at $275.0 \mathrm{eV}$, below the $\mathrm{C} 1 \mathrm{~s}$ edge. Identical results were obtained using a PS on SiN sample, indicating negligible overall contributions from the SiN substrate.

The estimated critical dose for the $\mathrm{C} 1 \mathrm{~s}(\mathrm{C}=\mathrm{C}) \rightarrow \pi^{*}{ }_{(\mathrm{C}=\mathrm{C})}$ transition at $285.1 \mathrm{eV}$ in PS is 1200(180) MGy [9]. It is so high that carbon contamination in the STXM vacuum chamber $[6,10,39]$ prevented us from performing the same C 1s NEXAFS spectral feature decay experiment used for PMMA in §3.1. Essentially, at higher dose more carbon is deposited onto the irradiated area, and the C 1s NEXAFS signal from the irradiated area becomes convoluted with the $\mathrm{C}$ 1s NEXAFS signal of the deposited carbon leading to a systematic error. We found that this influence of beam deposited carbon on $\mathrm{C} 1 \mathrm{~s}$ NEXAFS spectra of PMMA and PS became non-negligible beyond 300 MGy. A lithography experiment was also performed for PS. $600 \mathrm{~nm} \times 600 \mathrm{~nm}$ areas of a PS on SiN sample were irradiated at three photon energies: $275.0 \mathrm{eV}$ (below the $\mathrm{C} 1 \mathrm{~s}$ edge), $285.1 \mathrm{eV}$ (at the $\left.\mathrm{C} 1 \mathrm{~s}(\mathrm{C}=\mathrm{C}) \rightarrow \pi^{*}{ }^{(} \mathrm{C}=\mathrm{C}\right)$ transition) and $300.0 \mathrm{eV}$ (above the $\mathrm{C} 1 \mathrm{~s}$ edge) over a range of doses. The adsorbed dose rates for these exposures varied between $25-450$ MGy/s. The irradiated areas were not imaged using STXM after exposure. Instead, the sample was removed from the STXM chamber and underwent the PS development procedure (§2.3). The developed irradiated areas were imaged using AFM (Fig. 5(a)). 
(a)
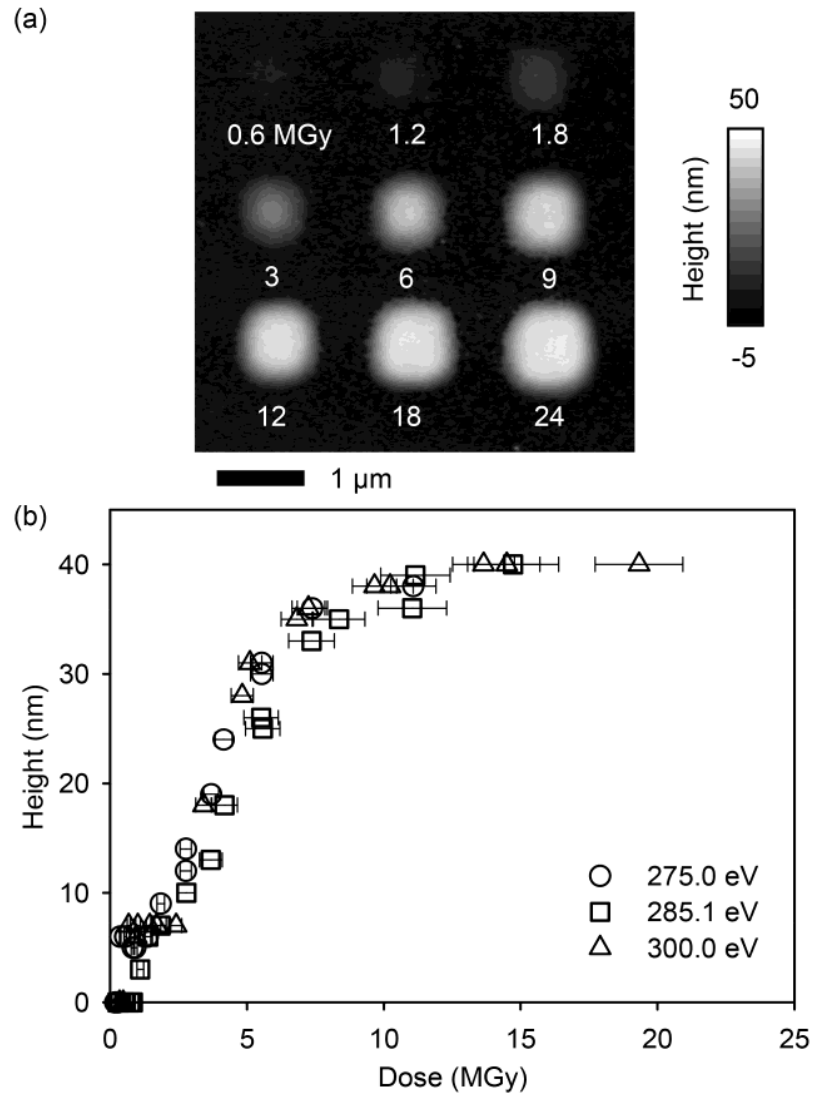

Figure 5: (a) Atomic force micrograph of irradiated areas of a $40 \pm 3 \mathrm{~nm}$ thick PS sample after

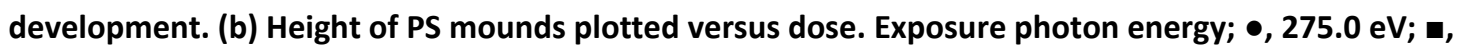
$285.1 \mathrm{eV} ; \Delta, 300.0 \mathrm{eV}$.

We observed that PS is a negative mode resist with this developer, with an onset of crosslinking of $300 \pm 100 \mathrm{kGy}$. The height of the mounds of cross-linked material in the developed irradiated areas increased with dose, up to about 15 MGy where the height reached a maximum value of $40 \pm 5 \mathrm{~nm}$, identical to the virgin film thickness. This maximum value did not change up to $208 \mathrm{MGy}$, the highest dose investigated for this experiment. Comparing the morphology of negative mode PS to negative mode PMMA, the cross-linked areas are smooth and extend further laterally, which is consistent with observations from electron beam lithography [50]. The maximum height of each PS mound was measured using AFM and plotted versus dose for the three incident photon 
energies (Fig. 5(b)). We found that the height of the PS mounds depended on dose, but was independent of photon energy.

\section{DISCUSSION}

We designed our experiments so that excitation below the $\mathrm{C}$ 1s edge induced strictly valence excitation in the sample, and excitation on or above the $\mathrm{C}$ 1s edge induced mostly core excitations producing Auger cascades. The measured ratios of core excitation to valence excitation, derived from the measured C 1s NEXAFS spectrums of PMMA and PS (Figs. 1 and 4, respectively), are listed in Table 1.

Table 1: Ratio of the core shell (CS) signal to the underlying valence shell (VS) signal. The latter was obtained by extrapolation of the pre-C 1s edge signal, and was subtracted from the full spectrum to obtain the CS-only signal.

\begin{tabular}{|c|c|c|}
\hline Polymer & Photon energy (eV) & CS/VS ratio \\
\hline PMMA & 275.0 & 0 \\
\hline PMMA & 288.4 & 46 \\
\hline PMMA & 300.0 & 18 \\
\hline PS & 275.0 & 0 \\
\hline PS & 285.1 & 110 \\
\hline PS & 300.0 & 50 \\
\hline
\end{tabular}

Given an identical dose (i.e., identical amount of energy absorbed per unit mass) below, on and above the $\mathrm{C}$ 1s edge, we found no change in radiation damage yields in PMMA and PS as measured by C 1s NEXAFS spectroscopy and lithography. The ratio of selective core excitation to valence excitation was as high as 110 (Table 1), but this had no effect on net damage yields. These results confirm that the absorbed dose is what 
determines the amount of damage, not the photon energy or the occurrence of Auger events. We found that both PMMA and PS could be damaged at $275.0 \mathrm{eV}$, which is below the $\mathrm{C}$ 1s edge, and that chemical changes induced below and above the $\mathrm{C} 1 \mathrm{~s}$ onset were similar (as judged by spectral changes). These results clearly show that Auger events are not required to damage PMMA or PS. The sensitivity of PMMA and PS was observed to be achromatic for $\mathrm{x}$-ray exposure across the $\mathrm{C}$ 1s edge, regardless of the very different electronic excitation and relaxation processes that occur below and above the onset of core level excitation [20].

It is difficult, if not impossible, to use negative results such as these to categorically deny the existence of an incident energy threshold for radiation damage rooted in valence versus core excitation. It may be that certain samples or signals do show an energy dependence while others do not. For example, cryo-cooling stabilizes diffraction contrast in x-ray crystallography [8], and prevents mass loss from occurring in electron microscopy [12], thus it is judged as an effective damage reduction strategy. But cryocooling does not affect the rate of chemical bond change measured by O 1s NEXAFS spectroscopy [13], and by this measure it is not effective. It may also be the case that dose accuracy better than $10 \%$ is required to observe the damage threshold effect (if so, it is likely of very limited practical use). Comparing our results with previous observations of a damage threshold in electron-impact ionization [37,38], we conclude that the reduction in damage observed for beam energies below $1000 \mathrm{eV}$ is related to the increase in stopping power at very low electron impact energies. Further studies using $\mathrm{x}$-rays with proper samples (e.g., known composition, homogeneous, uniform thickness), tight control on all variables (e.g., absence of air, water), and measuring the influence of 
possible sources of systematic error (e.g., dose rate, carbon contamination, beam induced temperature rise, correction for mass loss) are needed to confirm if this incident energy threshold for radiation damage exists at all. Questions about how to exploit the hypothesized damage threshold can be addressed at a later date.

\section{CONCLUSION}

Experiments were performed to resolve the question of an incident energy threshold for radiation damage rooted in valence electron versus core electron excitation. No significant difference in the dose dependence of the radiation damage was observed for either poly(methyl methacrylate) or polystyrene for excitation energies below the C 1s edge, at the most prominent $\mathrm{C} 1 \mathrm{~s} \rightarrow \pi^{*}$ resonant feature or above the $\mathrm{C} 1 \mathrm{~s}$ edge. Instead, the radiation-induced changes to these organic polymers were proportional to absorbed dose and independent of photon energy within the measurement uncertainty of $10 \%$. Radiation damage to poly(methyl methacrylate) or polystyrene did not require $\mathrm{C} 1 \mathrm{~s}$ excitation; no energy dependent damage thresholds were observed.

\section{ACKNOWLEDGEMENTS}

This research was supported by NSERC and the Canada Research Chairs Program. We thank Dr. David Kilcoyne and Dr. Tolek Tyliszczak at ALS 5.3.2.2, and Dr. Jian Wang and Dr. Chithra Karunakaran at CLS 10ID-1 for support and maintenance of the respective beamlines. The ALS is supported by the Director, Office of Energy Research, Office of Basic Energy Sciences, Materials Sciences Division of the U.S. Department of Energy under Contract No. DE-AC02-05CH11231. The CLS is supported by NSERC, 
CIHR, NRC and the University of Saskatchewan. AFGL acknowledges receipt of an ALS doctoral fellowship in residence and the CLS graduate student travel support program.

\section{REFERENCES}

1. M.R. Howells, T. Beetz, H.N. Chapman, C. Cui, J.M. Holton, C.J. Jacobsen, J. Kirz, E. Lima, S. Marchesini, H. Miao, D. Sayre, D.A. Shapiro, J.H.C. Spence, D. Starodub, J. Electron Spectrosc. Relat. Phenom. 170 (2009) 4-12.

2. R.F. Egerton, Ultramicroscopy 127 (2013) 100-108.

3. V.V. Voevodskii, Y.N. Molin, Rad. Res. 17 (1962) 366-378.

4. M. S. Isaacson, Inelastic scattering and beam damage of biological molecules, in: B.M. Siegel, D.R. Beaman (Eds.), Physical Aspects of Electron Microscopy and Microbeam Analysis, John Wiley \& Sons, New York, 1975, Chap. 14.

5. V.E. Cosslett, J. Microsc. Spectrosc. Electron. 3 (1978) 551-562.

6. R.F. Egerton, P. Li, M. Malac, Micron 35 (2004) 399-409.

7. R.F. Egerton, Electron Energy Loss Spectroscopy in the Electron Microscope, third ed., Springer, New York, 2011.

8. E.F. Garman, Acta Crystallogr. D66 (2010) 339-351.

9. J. Wang, C. Morin, L. Li, A.P. Hitchcock, A. Scholl, A. Doran, J. Electron Spectrosc. Rel. Phenom. 170 (2009) 25-36.

10. A.F.G. Leontowich, A.P. Hitchcock, J. Vac. Sci. Technol. B 30 (2012) 030601.

11. R. Henderson, Proc. R. Soc. B. 241 (1990) 6-8.

12. R.F. Egerton, Ultramicroscopy 5 (1980) 521-523. 
13. T. Beetz, C. Jacobsen, J. Synchrotron Rad. 10 (2003) 280-283.

14. H.N. Chapman, A. Barty, M.J. Bogan, S. Boutet, M. Frank, S.P. Hau-Riege, S. Marchesini, B.W. Woods, S. Bajt, W.H. Benner, R.A. London, E. Plönjes, M. Kuhlmann, R. Treusch, S. Düsterer, T. Tschentscher, J.R. Schneider, E. Spiller, T. Möller, C. Bostedt, M. Hoener, D.A. Shapiro, K.O. Hodgson, D. van der Spoel, F. Burmeister, M. Bergh, C. Caleman, G. Huldt, M.M. Seibert, F.R.N.C. Maia, R.W. Lee, A. Szöke, N. Timneanu, J. Hajdu, Nat. Phys. 2 (2006) 839-843.

15. A. Barty et al., Nat. Photonics 6 (2012) 35-40.

16. D.E. Johnson, Rad. Res. 49 (1972) 63-84.

17. M. Isaacson, J. Chem. Phys. 56 (1972) 1813-1818.

18. J. Durup, R.L. Platzman, Int. J. Radiat. Phys. Chem. 7 (1975) 121-136.

19. S.G. Urquhart, H. Ade, J. Phys. Chem. B 106 (2002) 8531-8538.

20. A.P. Hitchcock, J.J. Neville, in: T.-K. Sham (Ed.), Chemical Applications of Synchrotron Radiation, Part I: Dynamics and VUV Spectroscopy, World Scientific, Singapore, 2002, pp. 154-227.

21. B.J. Lin, J. Vac. Sci. Technol. 12 (1975) 1317-1320.

22. R.F. Egerton, R. McLeod, F. Wang, M. Malac, Ultramicroscopy 110 (2010) 991-997.

23. B. Fayard, A. Touati, F. Abel, M.A. Herve du Penhoat, I. Despiney-Bailly, F. Gobert, M. Ricoul, A. L’Hoir, M.F. Politis, M.A. Hill, D.L. Stevens, L. Sabatier, E. Sage, D.T. Goodhead, A. Chetioui, Rad. Res. 157 (2002) 128-140.

24. D.T. Goodhead, Rad. Res. 91 (1982) 45-76. 
25. K. Kobayashi, K. Hieda, H. Maezawa, Y. Furusawa, M. Suzuki, T. Ito, Int. J. Radiat. Biol. 59 (1991) 643-650.

26. L. Chen, J. Yan, S. Jiang, W. Zhang, Rad. Protect. Dosim. 133 (2009) 20-24.

27. T.-K. Sham, Chemical Applications of Synchrotron Radiation. Part II: X-ray Applications, World Scientific, Singapore, 2002, pp. 1005.

28. N. Shimizu, K. Hirata, K. Hasegawa, G. Ueno, M. Yamamoto, J. Synchrotron Rad. 14 (2007) 4-10.

29. T. Coffey, S.G. Urquhart, H. Ade, J. Electron. Spectrosc. Rel. Phenom. 122 (2002) $65-78$.

30. A. Yokoya, R. Watanabe, T. Hara, J. Radiat. Res. 40 (1999) 145-158.

31. K. Fujii, A. Yokoya, Rad. Phys. Chem. 78 (2009) 1188-1191.

32. J. Wang, G.A. Botton, M.M. West, A.P. Hitchcock, J. Phys. Chem. B 113 (2009) 1869-1876.

33. K. Hieda, T. Hirono, A. Azami, M. Suzuki, Y. Furusawa, H. Maezawa, N. Usami, A. Yokoya, K. Kobayashi, Int. J. Radiat. Biol. 70 (1996) 437-445.

34. D.M. Hanson, S.L. Anderson, M.C. Nelson, G.P. Williams, N. Lucas, J. Phys. Chem. 89 (1985) 2235-2237.

35. M.C.K. Tinone, K. Tanaka, J. Maruyama, N. Ueno, M. Imamura, N. Matsubayashi, J. Chem. Phys. 100 (1994) 5988-5995.

36. E. Ikenaga, K. Isari, K. Kudara, Y. Yasui, S.A. Sardar, S. Wada, T. Sekitani, K. Tanaka, K. Mase, S. Tanaka, J. Chem. Phys. 114 (2001) 2751-2759. 
37. A. Howie, F.J. Rocca, U. Valdre, Phil. Mag. B. 52 (1985) 751-757.

38. M.R. Stevens, Q. Chen, U. Weierstall, J.C.H. Spence, Microsc. Microanal. 6 (2000) 368-379.

39. A.F.G. Leontowich, A.P. Hitchcock, T. Tyliszczak, M. Weigand, J. Wang, C. Karunakaran, J. Synchrotron Rad. 19 (2012) 976-987.

40. M.Y. Efremov, S.S. Soofi, A.V. Kiyanova, C.J. Munoz, P. Burgardt, F. Cerrina, P.F. Nealey, Rev. Sci. Instrum. 79 (2008) 043903.

41. A.L.D. Kilcoyne, T. Tyliszczak, W.F. Steele, S. Fakra, P. Hitchcock, K. Franck, E. Anderson, B. Harteneck, E.G. Rightor, G.E. Mitchell, A.P. Hitchcock, L. Yang, T. Warwick, H. Ade, J. Synchrotron Rad. 10 (2003) 125-136.

42. K.V. Kaznatcheev, Ch. Karunakaran, U.D. Lanke, S.G. Urquhart, M. Obst, A.P. Hitchcock, Nucl. Instrum. Methods Phys. Res. A 582 (2007) 96-99.

43. R. Sanishvili, D.W. Yoder, S.B. Pothineni, G. Rosenbaum, S. Xu, S. Vogt, S. Stepanov, O.A. Makarov, S. Corcoran, R. Benn, V. Nagarajan, J.L. Smith, R.F. Fischetti, Proc. Nat. Acad. Sci. U.S.A. 108 (2011) 6127-6132.

44. R.F. Egerton, S. Lazar, M. Libera, Micron 43 (2012) 2-7.

45. K. Siangchaew, M. Libera, Phil. Mag. A 80 (2000) 1001-1016.

46. S. Tanuma, C.J. Powell, D.R. Penn, Surf. Interf. Anal. 21 (1993) 165-176.

47. M.O. Krause, J. Phys. Chem. Ref. Data 8 (1979) 307-327.

48. A.F.G. Leontowich, A.P. Hitchcock, Analyst 137 (2012) 370-375.

49. I. Haller, M. Hatzakis, R. Srinivasan, IBM J. Res. Dev. 12 (1968) 251-256. 
50. H.Y. Ku, L.C. Scala, J. Electrochem. Soc. 116 (1969) 980-985.

51. A.F.G. Leontowich, A.P. Hitchcock, Appl. Phys. A 103 (2011) 1-11.

\section{TABLE CAPTIONS}

TABLE 1. Ratio of the core shell (CS) signal to the underlying valence shell (VS) signal.

The latter was obtained by extrapolation of the pre-C 1s edge signal, and was subtracted from the full spectrum to obtain the CS-only signal.

\begin{tabular}{|c|c|c|}
\hline Polymer & Photon energy (eV) & CS/VS ratio \\
\hline PMMA & 275.0 & 0 \\
\hline PMMA & 288.4 & 46 \\
\hline PMMA & 300.0 & 18 \\
\hline PS & 275.0 & 0 \\
\hline PS & 285.1 & 110 \\
\hline PS & 300.0 & 50 \\
\hline
\end{tabular}




\section{FIGURE CAPTIONS}

FIG 1. C 1s NEXAFS spectrum of a $50 \pm 3 \mathrm{~nm}$ thick film of PMMA.

FIG 2. (a) Transmission x-ray micrograph, acquired at $288.4 \mathrm{eV}\left(\mathrm{C} 1 \mathrm{~s}(\mathrm{C}=\mathrm{O}) \rightarrow \pi^{*} \mathrm{C}=\mathrm{O}\right.$ peak) and converted to optical density, depicting nine areas of a $50 \pm 3 \mathrm{~nm}$ thick PMMA sample that were exposed to variable doses (values indicated). (b) Optical density at $288.4 \mathrm{eV}$ of irradiated areas of the sample plotted versus dose. Exposure photon energy: $\bullet, 275.0 \mathrm{eV} ; \mathbf{\square}, 288.4 \mathrm{eV} ; \boldsymbol{\Delta}, 300.0 \mathrm{eV}$.

FIG 3. Atomic force micrographs of irradiated areas of a $50 \pm 3 \mathrm{~nm}$ thick PMMA sample after development. Exposure photon energy: (a), $275.0 \mathrm{eV}$; (b), $288.4 \mathrm{eV}$; (c), $300.0 \mathrm{eV}$. All micrographs are on the same height and spatial scale.

FIG 4. C 1s NEXAFS spectrum of a $40 \pm 3 \mathrm{~nm}$ thick film of PS (black), and after receiving $70 \mathrm{MGy}$ at $275.0 \mathrm{eV}$ (red). The inset shows the reduction and slight blue shift in the $\mathrm{C} 1 \mathrm{~s}(\mathrm{C}=\mathrm{C}) \rightarrow \pi^{*} \mathrm{C}=\mathrm{C}$ peak due to the $70 \mathrm{MGy}$ exposure.

FIG 5. (a) Atomic force micrograph of irradiated areas of a $40 \pm 3 \mathrm{~nm}$ thick PS sample after development. (b) Height of PS mounds plotted versus dose. Exposure photon energy: •, $275.0 \mathrm{eV} ; \boldsymbol{\bullet}, 285.1 \mathrm{eV} ; \boldsymbol{\Delta}, 300.0 \mathrm{eV}$. 